\title{
Das Asienbeet - ein neuer Themengarten mit anspruchsvollen Zöglingen
}

\author{
SVEn NÜRNBERgER
}

\begin{abstract}
A new Asiatic Garden has been established in the southeastern section of the Palmengarten. In early 2010 a partially shaded area of $300 \mathrm{~m}^{2}$ was conceptionally redesigned to serve as a display for unique herbaceous perennials originating from different altitudes within the Himalayans and adjoining territories.
\end{abstract}

\section{Zusammenfassung}

Im südöstlich gelegenen Schattengartenareal des Palmengartens begannen im Frühjahr 2010 die Gestaltungsarbeiten für einen neuen Schaugartenbereich mit Pflanzen aus dem asiatischen Raum. Auf einer Fläche von etwa $300 \mathrm{~m}^{2}$ entstand eine artenreiche Staudenpflanzung mit unterschiedlichen Lebensbereichen.

\section{Einführung}

Der hohe Himalaya und die angrenzenden gemäßigten Zonen Ostasiens sind die Heimat und häufig auch das Diversitätszentrum vieler bekannter Staudengattungen. Das neue Asienbeet bietet einen besonderen Einblick in diese vielfältige Pflanzenwelt hoher Berge und tiefer Täler. Den Schwerpunkt bilden Arten aus kühlen montanen bis alpinen Höhenlagen, zudem werden auch Pflanzen aus klimamilden Zonen gezeigt, die im Frankfurter Klima mit entsprechenden Schutzmaßnahmen auch kalte Winter überstehen, wie z. B. verschiedene Gesneriaceen. Die Vergesellschaftung der Arten erfolgte nur ansatzweise pflanzensoziologisch. Sie orientiert sich vielmehr an der Möglichkeit unterschiedliche Standorttypen darzustellen. Die klimatischen Bedingungen im Frankfurter Palmengarten sowie die besondere Topographie des Asienbeetes laden zu einer derartigen Konzeption ein und lassen dem gärtnerisch-gestalterischen Konzept einen großen Spielraum, fordern aber auch zu unkonventionellen Überlegungen heraus. Was bedeutet das für die Planung und Ausführung?

\section{Pflanzfläche}

$\mathrm{Zu}$ Anfang der Planung wurde die Pflanzfläche von 40 x 7 (-8) Metern genauer unter die Lupe genommen. Bodenverhältnisse, Lichtintensität, Geländeneigung und die vorhandene Vegetationsdecke wurden bewertet. Daraus ergaben sich zunächst fünf unterschiedliche Lebensbereiche mit Lichtverhältnissen von voll- bis absonnig, lichtem Schatten bis zu vollschattigen Bereichen. Aufgrund der integrierten Baumbestände aus Fagus sylvatica und Crataegus monogyna und einer leichten Neigung des Geländes weist der Boden unterschiedliche Feuchtebereiche auf. Angestrebt wurde die Bildung weiterer Lebensbereiche, die miteinander fließend verknüpft werden sollten. Durch die hälftige Trennung der Beetfläche mit einem Gesteinsband in der nördlichen bis zentralen Flucht entstanden zwei Ebenen mit unterschiedlichen Feuchtebereichen und verschiedene Pflanznischen mit einem speziellen Kleinklima. Die Gesteinskette besteht aus einem Granodiorit, welcher nahe der Grube Messel am Rande des Odenwaldes ansteht. Das Gestein zeichnet sich durch ein auffälliges Farbenspiel mit rötlichen Tönen aus, das besonders bei nasser Oberfäche zur Geltung kommt. Das Gesteinsband stabilisiert die obere Terrasse mit abwechslungsreichen Abschnitten, die einer natürlichen Ablagerung von Gesteinsmassen im Gebirge nachempfunden ist. Schutthalde, lockeres Hanggestein und eine kleine Verwerfung stehen im Wechsel. Aufgrund der unterschiedlichen Licht- und Feuchteverhältnisse in dieser Beetzone konnten verschiedene Lebensbereiche geschaffen werden (vgl. NürnbergeR 2012). Der vollsonnige Abschnitt bietet die Möglichkeit montane Wiesenpflanzen und 
Stauden offener weiträumiger Standorte zu thematisieren. Hier erkennt der Gartenliebhaber die Eltern einiger Prachtstaudensorten wieder, z. B. Hemerocallis-, Delphinium-, Lilium- und Geranium-Arten. Die Beetmitte wird durch eine Senke mit Feuchtwiesen- und Bachlaufpflanzen geprägt. Hier dominieren im Spätfrühjahr Trollblumen und Iris-Arten das Bild. Durch die Kapillarwirkung des feuchten Randbereichs und den Übergang zu absonnigen Lichtverhältnissen entstand hier ein kühlfeuchtes Milieu, das besonders den Pflanzen aus Höhenlagen zwischen $3500 \mathrm{~m}$ und $5000 \mathrm{~m}$ zu Gute kommt. Zahlreiche Primelarten, blaue Lerchensporne und Meconopsis-Arten profitieren von der geringen Aufheizung des Standortes während des Hochsommers. An dieser Stelle läuft das Gesteinsband flach aus und bildet den Übergang zum lichten Schatten. Dieser wird von Pflanzenarten bestimmt, die ihren Ursprung in montanen Wäldern und kühlen Schluchten Westchinas, Koreas und Japans haben. Der Randbereich vergesellschaftet niedrige Frühlingsblüher, die im Verlauf von spätaustreibenden Ingwergewächsen und Aronstabgewächsen abgelöst werden. Bekannte Blattschmuckstauden wie Hosta plantaginea und unterschiedlichste Farne eröffnen den Übergang zu imposanten Großstauden und einigen sehenswerten Gehölzen, wie die erst kürzlich beschriebene Ebereschenart Sorbus rosea aus Pakistan.

\section{Die Pflanzenwahl}

Zu Beginn der Planung wurde eine Bestandsaufnahme erstellt, welche asiatischen Pflanzenarten aktuell im Palmengarten zur Verfügung stehen und in das künftige Konzept übernommen werden könnten (gute Anregungen bei

Abb. 1 (oben): Die Fläche neben dem Caféhaus Siesmayer vor der Bearbeitung im Herbst 2009.

Abb. 2 (Mitte): Vorbereitungen für die neue Pflanzung und die neu gesetzten Steine im Sommer 2010.

Abb. 3 (unten): Schattiger Bereich unter den älteren Gehölzen.
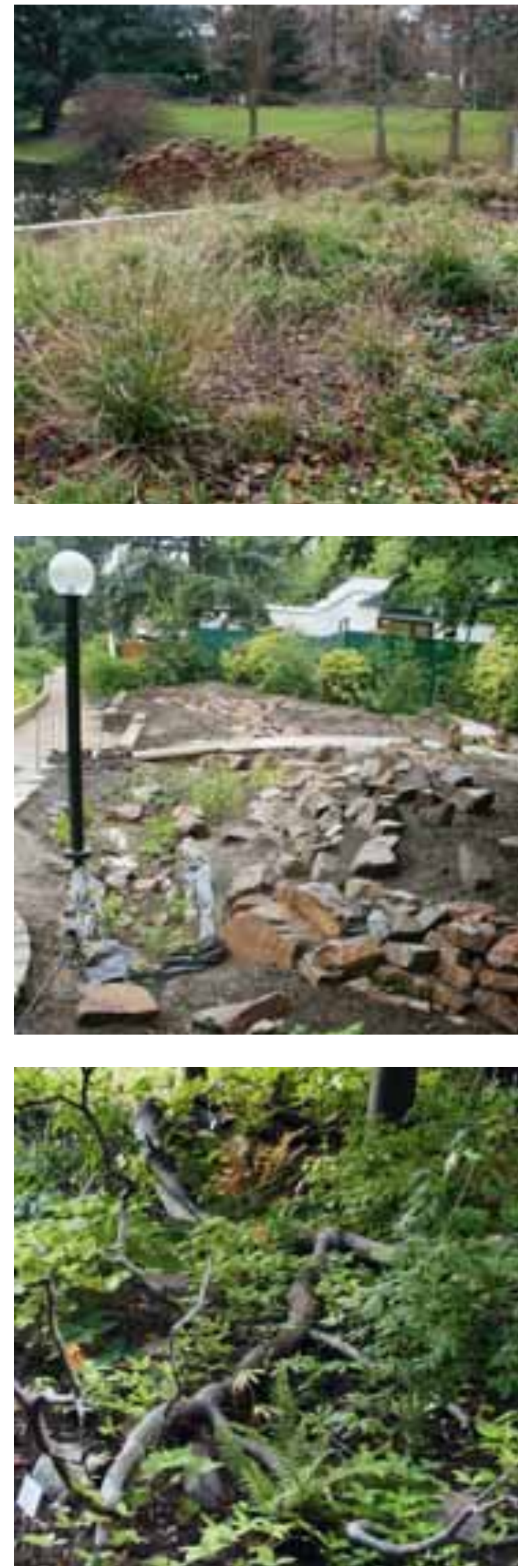

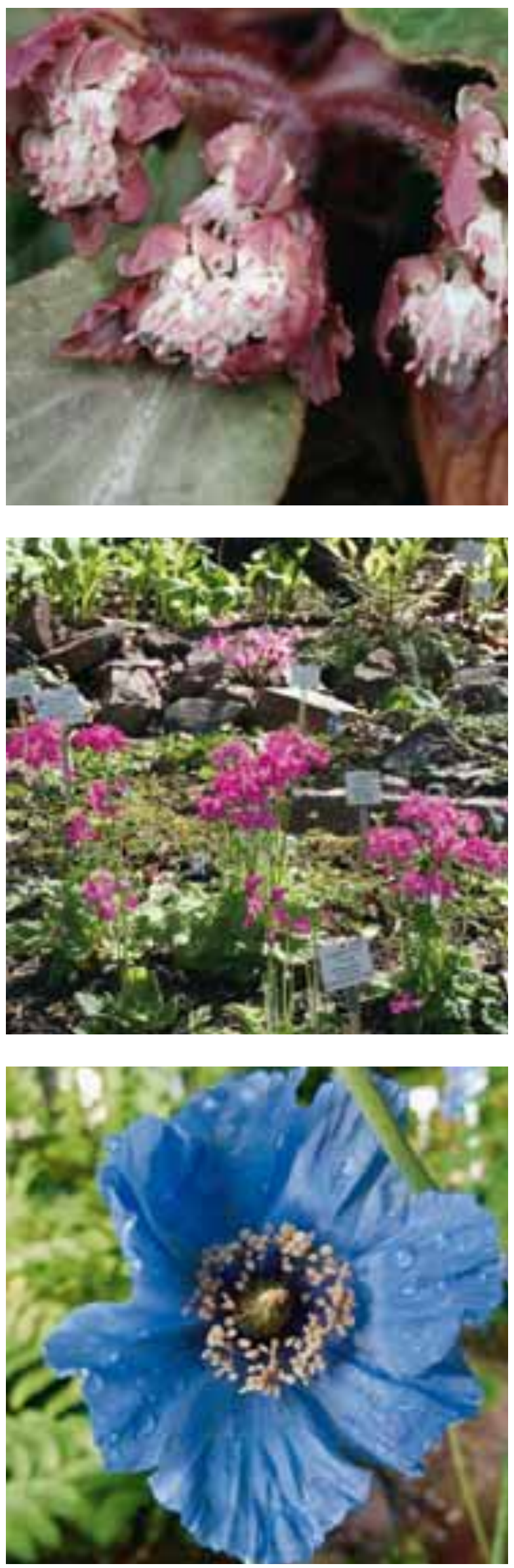

JERMYN 2001). Danach galt es Vergesellschaftungen zu finden, die die floristische Vielfalt dieser weiträumigen geografischen Areale widerspiegeln unter der Maßgabe, ein Artenspektrum zu verwenden, das dauerhaft unter den hiesigen Standortbedingungen funktionieren würde. Die Beschaffung von Pflanzenmaterial in Form von Lebendpflanzen und Saatgut über das Netzwerk Botanischer Gärten und der $\mathrm{Zu}$ kauf einiger Arten ermöglichte eine zeitnahe Komplettierung der Bepflanzung der Anlage. An dieser Stelle sei der jährlichen Samentauschaktion der Botanischen Gärten gedankt, sowie insbesondere den Botanischen Gärten Heidelberg, München, Darmstadt und Lyon für die Bereitstellung von Lebendmaterial.

\section{Gattungsschwerpunkte}

Die unterschiedlichen Mikroklimate des Themengartens ermöglichen die Verwendung verschiedener Schwerpunktgattungen mit einem breiteren Artenspektrum. Dies gibt Raum für die Darstellung von ökologischen Anpassungen unterschiedlicher Arten innerhalb einer Gattung. Gärtnerisch ist damit auch eine Staffelung der Blütezeiten verbunden. Die Gattung Primula eignet sich zu diesem Zwecke besonders gut. So beginnt das Primeljahr im Asienbeet Anfang April mit der farbenprächtigen Primula rosea und den cremefarbenen Glöckchen von Primula sikkimensis var. hopeana am Rande des dauerfeuchten Caltha-Sumpfes. Unmittelbar darauf folgen Primula cortusoides, $P$. involucrata, die kaukasische $P$. aurantiaca und $P$. dickieana im durchlässigeren aber dennoch feuchten Boden. Besonders eindrucksvoll schieben sich dann ab Mai bis in den Juni Primula alpicola, P. secundiflora, P. burmanica und die „Orchideenprimel“ $P$. vialii empor. Im

Abb. 4 (oben): Zu den ersten Blühern des Jahres gehört im Asienbeet eine eher unbekannte Milzkraut-Art (Chrysosplenium macrophyllum).

Abb. 5 (Mitte): Seit der Anlage des Beetes hat sich Primula cortusoides bereits gut spontan vermehrt.

Abb. 6 (unten): Meconopsis horridula. 
Spätfrühling öffnen die ersten Krötenlilien (Tricyrtis) die Blütenstände und blühen in verschiedenen Arten nach und nach bis in den Herbst hinein. Ausgesprochen attraktiv sind gerade auch die gelbblühenden Tricyrtis-Arten, deren Blüteninneres rötlich oder bräunlich gefleckt sein kann.

\section{Jahreszeitlicher Verlauf}

Schaupflanzungen können von der Präsentation seltener oder ungewöhnlicher Pflanzenarten profitieren, locken den Betrachter aber auch besonders mit saisonalen Blüh- und Blattschmuckaspekten. Im Asienbeet können die ersten Blüten bei milder Wetterlage schon während des Winters erscheinen, wenn das chinesische Milzkraut Chrysosplenium macrophyllum seine anmutigen Blütenstände nach oben schiebt, gefolgt von den skurrilen Aasblüten der gefleckten Asarum-Arten und den hübschen Glöckchen von Disporum uniflorum. Im Mai beginnt dann die Zeit der blauen HimalayaBlüten (vgl. auch Jürgens 2009). Meconopsis horridula und $M$. betonicifolia-Hybriden stehen mit Corydalis elata und C. flexuosa in den Blütenflecken der schon erwähnten Primelarten. Kräftig pinkfarbene Freilandgloxinien schießen wie Pilze täglich aus dem Boden und harmonieren farblich mit dem Gelb der Zwergtrollblumen. Im Juni dominieren zunächst die großen Trollblumen und verschiedene Iris- und Geranium-Arten. Delphinium cashmerianum und $D$. tatsiense, die zu den halbhohen Ritterspornen gehören, leiten die Welle der Frühsommerblüher ein. Lilium regale, $L$. henryi und L. sargentiae verwandeln nun den sonnigen Bereich in ein Trompetenorchester, während im schattigen Areal Lilium hansonii schon einige Tage zuvor die goldgelben Blüten geöffnet hat.

Abb. 7 (oben): Pleione limprichtii, eine bei uns winterharte Erdorchidee.

Abb. 8 (Mitte): Paeonia rockii, benannt nach dem in Asien aktiven Pflanzensammler Joseph Rock.

Abb. 9 (unten): Codonopsis lanceolata, ein kletterndes Glockenblumengewächs.
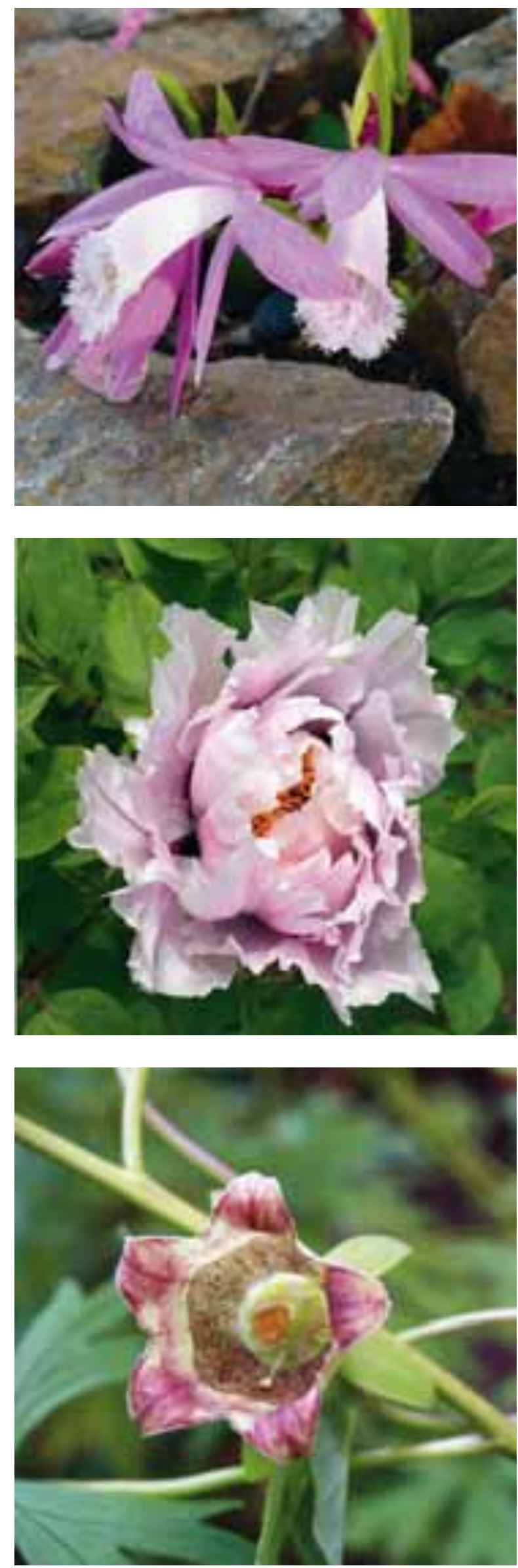

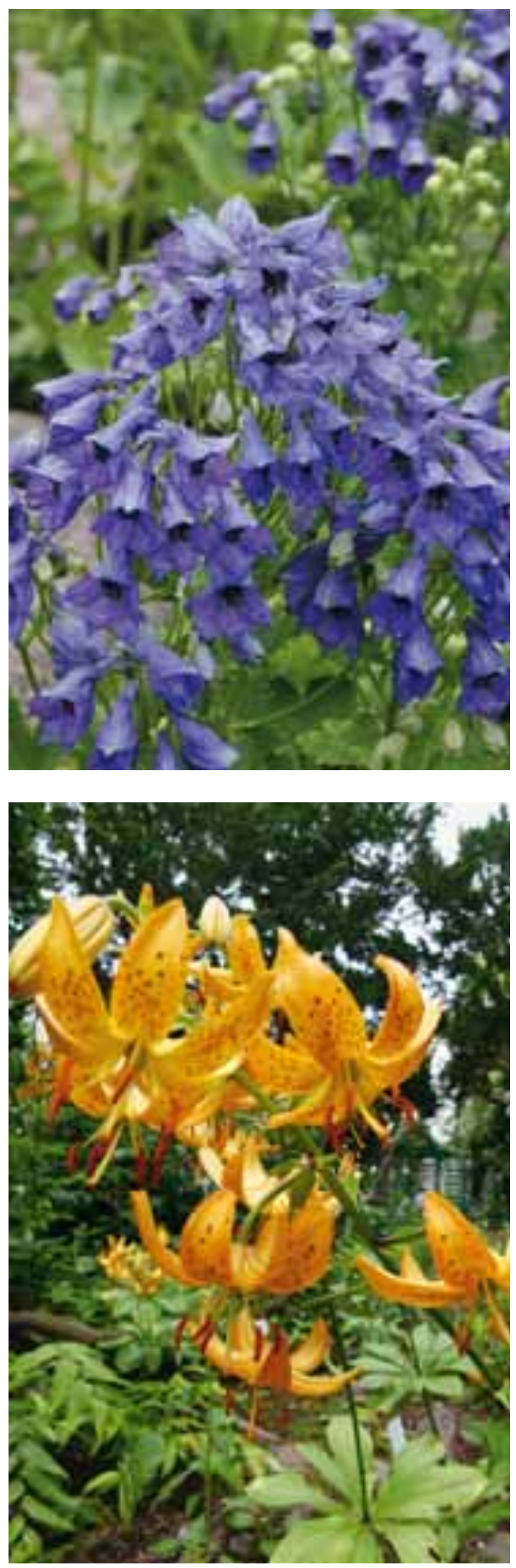

Hier winden sich auch einige bemerkenswerte Kletterstauden empor: Gattungen, deren Vertreter üblicherweise durch aufrecht wachsende Arten bekannt sind. Genannt seien an dieser Stelle Aconitum alboviolaceum und Dactylocapnos scandens (syn. Dicentra), ein gelbblühendes kletterndes Tränendes Herz. Zu den besonders interessanten Klettergewächsen Asiens gehören die Tigerglocken aus der Familie der Glockenblumengewächse. Codonopsis lanceolata ist eine der dekorativsten Arten und besticht durch eine farbenprächtige Zeichnung im Inneren der großen Blütenglocke. Blütenschleier im Hintergrund der Pflanzung werden nun durch verschiedene Astilbe-Arten und Thalictrum delavayi inszeniert. Im sonnigen Schutthang leuchten darauffolgend stachelige Kardendisteln mit kräftigen Rosatönen aus bläulichvioletten Geranium-Arten hervor. Der Spätsommer und Frühherbst wird durch auffällige Fruchtstände und erste Herbstfarben an Stauden und Gehölzen eingeleitet. Sinnlich stehen kerzenartige abgeblühte Infloreszenzen in den karminfarbenen Horsten von Geranium soboliferum und Sorbus setschwanensis zeigt schon als Jungpflanze, welche Farbwirkung in einigen Jahren den Standort bestimmen wird: purpurrote Blätter und weiße Früchte, vereint mit dem tiefen Blauviolett von Aconitum carmichaelii.

\section{Aussichten}

Pflanzungen sind dynamische Kompositionen, die im Laufe der Zeit Veränderungsprozessen unterliegen. Gärtnerisch kann man solche Abläufe steuern, kontrollieren und regulieren. So ist es notwendig, die Staudenpflanzungen durch Teilung und Maßnahmen der Bodenverbesserung dauerhaft vital zu halten. Neue Arten werden stetig Einzug in das Asienbeet halten, insbesondere Akzessionen vom Wildstandort, um die wissenschaftliche Wertigkeit für Forschungsarbeiten und Didaktik zu gewährleisten. Die gerüstbildenden Rotbuchen werden mit den Jahren größer und so die Standortbedingungen verändern. Es wird sich zeigen, welche Arten sich dauerhaft als praktikabel und robust behaupten werden und dem Wurzel- 
druck standhalten können. Und auch der schleichende Klimawandel hinterlässt mit ersten Wetterextremen Spuren - mit der Installation einer wassersparenden automatischen Bewässerungsanlage wurde bereits nachhaltig reagiert.

Das Asienbeet stellt eine gärtnerisch-kulturtechnische Herausforderung dar. Es ermöglicht spannende Langzeitstudien, bietet Gärtnern, Wissenschaftlern und Besuchern einen Schauplatz asiatischer Pflanzendiversität auf engstem Raum und lässt dabei die Grenzen zwischen Pflanzengeografie und Gartendesign verschmelzen.

\section{Dank}

Mein besonderer Dank gilt allen Kolleginnen und Kollegen, die sich mit großem Engagement für das Gelingen des Projektes „Asienbeet“ eingesetzt haben. Dank auch an alle, die sich durch inhaltliche Anregungen und Diskussionen dem Asienbeet gewidmet haben.

\section{Literatur}

Jermyn, J. 200I: The Himalayan Garden: Growing Plants from the Roof of the World. - Portland, Oregon. Jürgens, P. 2009: Scheinmohn im Garten und am Wildstandort. - Gartenpraxis 8/2009: 24-29.

Nürnberger, S. 2012: Wechselspiel zwischen Stein und Pflanze. Gartenpraxis 5/2012: 28-35.

Abb. 10 (Seite 64 oben): Delphinium cashmerianum dominiert im Frühsommer mit seinen kräftig blauen Blüten im sonnigen Teil des Asienbeets.

Abb. 11 (Seite 64 unten): Während die weißblütigen Lilien (Lilium regale, L. sargentiae) im Sommer im sonnigen Teil blühen, öffnet im Schattenbereich Lilium hansonii ihre orangegelben Blüten.

Abb. 12 (oben): Üppige rosaviolette Blütenpracht der Freilandgloxinien.

Abb. 13 (Mitte): Während trocken-heißer sommerlicher Witterung muss regelmäßig gewässert werden.

Abb. 14 (unten): Morina longifolia.
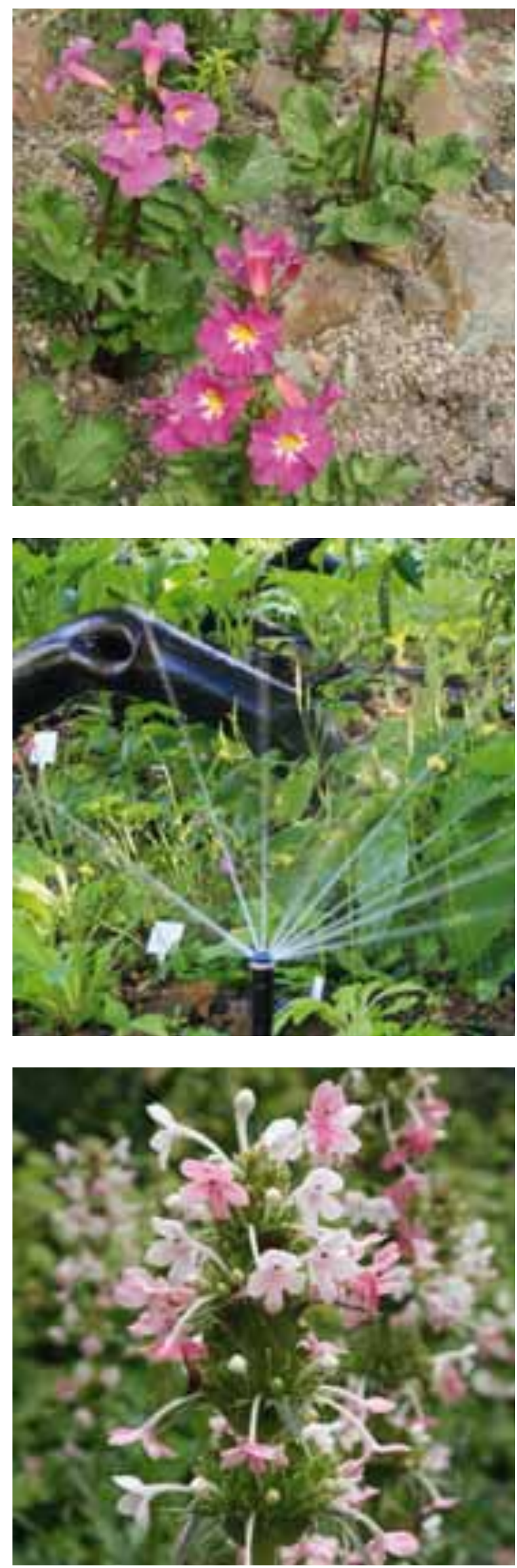